\title{
Physical health status assessed during hospitalization for acute coronary syndrome predicts mortality 12 months later ${ }^{23}$
}

\author{
Brett D. Thombs ${ }^{\mathrm{a}, *}$, Roy C. Ziegelstein ${ }^{\mathrm{b}}$, Donna E. Stewart ${ }^{\mathrm{c}, \mathrm{d}}$, Susan E. Abbey ${ }^{\mathrm{c}, \mathrm{d}}$, \\ Kapil Parakh ${ }^{\mathrm{b}}$, Sherry L. Grace ${ }^{\mathrm{c}, \mathrm{d}, \mathrm{e}}$
}

\begin{abstract}
Objective: Self-report measures of health status predict mortality in several groups of patients with cardiovascular disease, although overlap with symptoms of depression may reduce or eliminate this relationship. The association between self-reported health status and mortality has not been examined in patients hospitalized for acute coronary syndrome (ACS). The objective was to investigate whether the Physical Component Summary (PCS) and Mental Component Summary (MCS) scores of the SF12 predicted 12-month all-cause mortality after controlling for cardiac risk factors and symptoms of depression. Methods: The SF-12 and Beck Depression Inventory were administered 2-5 days after admission to $800 \mathrm{ACS}$ patients from 12 coronary care units. Logistic regression was used to assess the relationship of the PCS and MCS with mortality 12 months later, controlling for age, sex,
\end{abstract}

cardiac diagnosis (acute myocardial infarction vs. unstable angina), Killip class, history of myocardial infarction, and in-hospital depressive symptoms. Results: Lower scores on the SF-12 PCS (worse health) were associated with a significantly higher risk of mortality [odds ratio $(\mathrm{OR})=0.94,95 \%$ confidence interval $(C I)=0.92-0.97, P<.001]$. MCS scores failed to reach significance $(\mathrm{OR}=0.98, \mathrm{CI}=0.95-1.00, P=.053)$. The PCS significantly predicted mortality even after controlling for other cardiac risk factors and depressive symptoms ( $\mathrm{OR}=0.96, \mathrm{CI}=0.93-0.99, P=.008$ ), equivalent to a $34 \%$ increase in risk per 10 -point (1 SD) decrement in PCS scores. Conclusion: The brief SF-12 PCS presents an attractive option for improving risk stratification among hospitalized ACS patients.

Keywords: Acute coronary syndrome; Mortality; Quality of life; SF-12

This research was conducted with funds from the Heart and Stroke Foundation of Ontario and the Samuel Lunenfeld Foundation of Toronto, Ontario, awarded to Dr. Stewart and Dr. Abbey. Dr. Thombs is supported by a New Investigator Award from the Canadian Institutes of Health Research and an Établissement de Jeunes Chercheurs award from the Fonds de la Recherche en Santé Québec. Dr. Grace receives funding from the Canadian Institutes of Health Research, and Dr. Ziegelstein is supported by grant number R24AT004641 from the National Center for Complementary \& Altemative Medicine and by the Miller Family Scholar Program.

\section{Introduction}

Patient-reported health measures are increasingly being integrated into clinical practice and decision-making processes [1-3]. One reason for this is the recognition that "hard" outcomes like physiological measures may not always reflect all aspects of health. For example, pain, fatigue, depression, and ability to function are of great importance to patients but cannot be measured without patient evaluation. Global measures of health status have been designed to reflect health status and function as perceived by patients across domains of health that are 
important for patients [4]. For many patients with cardiovascular disease, health status is as important as survival [5]. Measures of patient-reported health status, such as the SF-36 [6] or the SF-12 [7], are widely used to evaluate the impact of a disease on patient well-being and as outcomes measures in studies of medical interventions. In addition, patient-reported health status may be useful as a prognostic or risk stratification tool to quantify underlying aspects of health not captured by overt indicators of disease severity or other standard risk factors [8].

Patient-reported health status has been shown to independently predict adverse outcomes, including mortality, in studies of patients following coronary artery bypass graft surgery [4], with stable coronary artery disease [9], and with heart failure [10-15]. Two studies of heart failure patients included symptoms of depression as a covariate, however, and both found that health status no longer predicted survival after controlling for depressive symptoms $[16,17]$. This is important because many studies have reported that depression is a significant predictor of mortality among patients with an acute coronary syndrome (ACS) [18-20]. The term ACS refers to an episode of acute myocardial ischemia, either with or without death or irreversible necrosis of heart muscle. ACS includes unstable angina (UA), in which myocardial ischemia is not severe or prolonged enough to cause detectable necrosis of heart muscle, and myocardial infarction (MI) in which detectable necrosis can be demonstrated. Acute myocardial infarction (AMI), in turn, is categorized based on the appearance of the electrocardiogram as either non-ST-segment elevation MI or ST-segment elevation MI [21]. No studies have investigated whether patient-reported health status assessed during hospitalization for ACS predicts post-discharge mortality, and, if so, whether it continues to predict mortality after controlling for prognostic variables that have been shown to affect survival, including symptoms of depression. The objective of this study was to investigate whether the Physical Component Summary and Mental Component Summary scores (PCS and MCS) of the SF-12 [7], a brief version of the widely used SF-36 [6], predicted 12-month all-cause mortality following ACS after controlling for other important risk factors, including symptoms of depression. We hypothesized that both the PCS and MCS would be significantly associated with mortality, but that the MCS would no longer be associated with mortality after controlling for depressive symptoms due to substantial overlap between the two variables.

\section{Methods}

\section{Patients and procedures}

Adult patients (18 years or older) who were diagnosed with an ACS (i.e., AMI or UA) were recruited by a research nurse on the second to the fifth day of their hospitalization.
This was a longitudinal observational study on depression and participation in cardiac rehabilitation that assessed patients in the hospital and by then at 6 and 12 months post-ACS by mail. Patients were recruited from 12 coronary care units in both large urban teaching hospitals and community hospitals in small- and medium-sized cities across Southcentral Ontario, Canada. Patients were excluded if they were medically unstable or unable to read or speak English. Eligible patients who agreed to participate gave informed, written consent and were provided with a selfreport questionnaire. All patients received standard care for their ACS. The original study protocol was approved by the Research Ethics Boards of the University of Toronto and University Health Network.

\section{Measures}

The SF-12 [7] was administered during the hospitalization, and the PCS and MCS were used to measure physical and mental health status, respectively. High scores on the PCS indicate better function, whereas low scores reflect important physical limitations, including difficulties in selfcare, physical and social activities, and role performance; high levels of pain; and/or fatigue [7]. Similarly, high scores on the MCS suggest the positive affect and absence of psychological distress, whereas low scores are indicative of poorer mental health, including high levels of psychological distress and social and role limitations due to emotional problems [7]. The SF-12 [7] is a 12-item version of the SF-36 [6]. A large longitudinal study of patients with coronary heart disease found that the SF-12 PCS and MCS were highly correlated with their respective summary scores on the SF-36 (both $r=.96, P<.001$ ). Change over time on the SF-36 PCS and MCS was also closely replicated by the SF12 PCS $(r=.95, P<.001)$ and MCS $(r=.94, P<.001)$ [22]. Raw scores on the SF-12 PCS and MCS are standardized to the general US population (mean $=50$, S.D. $=10$ ) to facilitate interpretation [7].

Symptoms of depression were assessed using the 21-item Beck Depression Inventory (BDI) [23]. Each item has four possible answers, scored $0-3$, indicating increasing symptom severity, and respondents are instructed to describe the way they have been feeling during the past week. The BDI is the most widely used measure of depressive symptoms in studies of patients with ACS [24].

Killip class [25], measured on a four-point scale, was used to indicate the presence of heart failure on admission to the hospital. Killip class is a simple clinical tool that has been found in many studies to predict mortality following ACS [26-28]. Killip class and history of previous AMI were determined from the medical record. Sociodemographic data and other health status variables, such as comorbidities and smoking status, were determined from the patient health questionnaire.

The primary outcome variable was all-cause mortality within 12 months of discharge from the hospital. For 
patients who did not return 12-month follow-up questionnaires, vital status was determined by contacting by telephone, in sequence as necessary, patients, patients' family members or contacts listed in the initial data collection form, patients' general practitioners, and patients' specialist physicians.

\section{Data analysis}

Patient demographic and medical data were compared between patients who died and patients who survived to 12 months post-discharge using the $\chi^{2}$ statistic for categorical variables and $t$ tests for continuous variables. The associations of the SF-12 PCS and MCS with 12-month mortality were assessed separately with logistic regression models, rather than with survival models, since survival status at 12 months was available, but data on time to death were not. For both the SF-12 PCS and MCS, the component summary score was initially entered alone, followed by a second model with known demographic and cardiac risk factors [age, sex, cardiac diagnosis (AMI vs. UA), history of MI, and Killip class], followed by a third model that added inhospital BDI scores. The set of covariates was limited to these variables based on consideration of the number of outcomes (mortality) being predicted. Additional covariates were added to the third model post hoc one at a time to determine whether they would have likely changed the association between the PCS or MCS and mortality. Based on the overall mortality rate of $5.6 \%$, the study had $80 \%$ power to detect an increase in mortality of $48 \%$ per decrease of 10 points ( $1 \mathrm{SD}$ in the normative sample) in PCS and MCS scores. Discrimination and calibration of the multivariate models were assessed based on the $c$-index and the HosmerLemeshow goodness-of-fit test statistic, respectively [29]. The $c$-index is the percentage of comparisons where patients who died had a higher predicted probability of death than patients who lived for all possible patient pairs, one of whom lived to 12 months post-discharge and the other of whom died by 12 months post-discharge [29]. Calibration was assessed using the Hosmer-Lemeshow goodness-of-fit test (HL) [29], which is a measure of the accuracy of the predicted number of deaths compared to the number of patients who actually die across the spectrum of probabilities. A relatively large $P$ value indicates that the model fits well and that there is not a large discrepancy between observed and expected mortality. All analyses were conducted using SPSS version 15.0 (SPSS, Inc, Chicago, IL, USA), and all statistical tests were two-sided with a $P<.05$ significance level.

\section{Results}

SF-12, sociodemographic, cardiac disease, and risk factor data were available from 812 patients in-hospital, and 800 had 12-month mortality data (45 deaths, $5.6 \%$ ). As shown in Table 1, patients who died within 12 months of discharge were significantly older, more likely to have high school

Table 1

Participants who were alive vs. those who died 12 months after discharge

\begin{tabular}{|c|c|c|c|c|}
\hline & All patients & Dead at 12 months & Alive at 12 months & $P$ value \\
\hline Total number & 800 & $45(5.6 \%)$ & $755(94.4 \%)$ & \\
\hline \multicolumn{5}{|l|}{ Sociodemographics } \\
\hline Age in years, mean \pm S.D. & $61.5 \pm 11.9$ & $68.3 \pm 11.1$ & $61.1 \pm 11.9$ & $<.001$ \\
\hline Male, $n$ & $533 / 800(66.6 \%)$ & $32 / 45(71.1 \%)$ & $501 / 755(66.4 \%)$ & .511 \\
\hline Family income $>$ Can $\$ 50,000, n$ & $310 / 688(45.1 \%)$ & $11 / 36(30.6 \%)$ & $299 / 652(45.9 \%)$ & .072 \\
\hline Married, $n$ & $593 / 791(75.0 \%)$ & $30 / 45(66.7 \%)$ & $563 / 746(75.5 \%)$ & .186 \\
\hline Education high school or less, $n$ & $541 / 782(69.2 \%)$ & $36 / 43(83.7 \%)$ & $505 / 739(68.3 \%)$ & .034 \\
\hline \multicolumn{5}{|l|}{ Health } \\
\hline Diagnosis of AMI, $n$ & $428 / 800(53.5 \%)$ & $24 / 45(53.3 \%)$ & $404 / 755(53.5 \%)$ & .982 \\
\hline Killip class $>\mathrm{I}, n$ & $126 / 800(15.8 \%)$ & $11 / 45(24.4 \%)$ & $115 / 755(15.2 \%)$ & .099 \\
\hline Peak creatinine kinase (IU), mean \pm S.D. ${ }^{a}$ & $1302 \pm 1507$ & $1328 \pm 1510$ & $825 \pm 1381$ & .128 \\
\hline Systolic blood pressure $>130 \mathrm{mmHg}, n$ & $459 / 796(57.7 \%)$ & $23 / 45(51.1 \%)$ & $436 / 751(58.1 \%)$ & .360 \\
\hline Diabetes mellitus, $n$ & $188 / 741(25.4 \%)$ & $14 / 43(32.6 \%)$ & $174 / 698(24.9 \%)$ & .264 \\
\hline Smoker, $n$ & $264 / 778(33.9 \%)$ & $14 / 44(31.8 \%)$ & $250 / 734(34.1 \%)$ & .760 \\
\hline Previous MI, $n$ & $228 / 800(28.5 \%)$ & $25 / 45(55.6 \%)$ & $203 / 756(26.9 \%)$ & $<.001$ \\
\hline History of CABG & $76 / 788(9.6 \%)$ & $6 / 45(13.3 \%)$ & $70 / 743(9.4 \%)$ & .430 \\
\hline History of PTCA & $42 / 788(5.3 \%)$ & $3 / 45(6.7 \%)$ & $39 / 743(5.2 \%)$ & .727 \\
\hline \multicolumn{5}{|l|}{ Physical function and depression } \\
\hline SF-12 PCS Score, mean \pm S.D. & $40.5 \pm 11.5$ & $33.2 \pm 9.8$ & $41.0 \pm 11.5$ & $<.001$ \\
\hline SF-12 MCS Score, mean \pm S.D. & $48.5 \pm 10.9$ & $45.5 \pm 11.4$ & $48.7 \pm 10.8$ & .051 \\
\hline BDI score, mean \pm S.D. & $8.7 \pm 7.4$ & $12.3 \pm 8.8$ & $8.5 \pm 7.3$ & .006 \\
\hline $\mathrm{BDI} \geq 10, n$ & $275 / 800(34.4 \%)$ & $23 / 45(51.1 \%)$ & $252 / 755(33.4 \%)$ & .015 \\
\hline
\end{tabular}

For certain items in the table, there are missing data as reflected in the denominators. For the SF-12 PCS and MCS scores, a higher score reflects better function. For

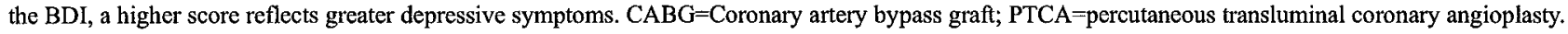

${ }^{\text {a }}$ Peak creatinine kinase reported only for patients with a diagnosis of AMI. 
education or less, and more likely to have had a previous MI. They rated their physical health status significantly worse based on the PCS and had more symptoms of depression. There was a nonsignificant trend for MCS scores to be lower and for Killip class to be $>1$ among patients who died within 12 months post-ACS. PCS scores among patients who died were 33.2 (1.68 SDs below the US general population mean) compared to 41.0 (0.9 SD below the mean for the US population) among patients alive at 12 months postdischarge. MCS scores for patients who died in the first 12 months post-discharge were 45.5 ( 0.45 SD below the US general population mean) compared to 48.7 ( $0.13 \mathrm{SD}$ below the US general population mean) for patients who survived to 12 months post-discharge. BDI scores were significantly correlated with both PCS $(r=-.33, P<.001)$ and MCS scores $(r=-.60, P<.001)$.

On a bivariate basis, lower scores on the SF-12 PCS were associated with a significantly higher risk of mortality [odds ratio $(\mathrm{OR})=0.94,95 \%$ confidence interval $(\mathrm{CI})=0.92-0.97$, $P<.001]$ (Table 2), whereas MCS scores failed to reach significance ( $\mathrm{OR}=0.98, \mathrm{Cl}=0.95-1.00, P=.053)$ (Table 3 ). The PCS continued to significantly predict 12-month mortality even after controlling for other demographic and cardiac risk factors and depressive symptoms $(\mathrm{OR}=0.96$, $\mathrm{CI}=0.93-0.99, P=.008$ ). This reflects risk per 1-point change in PCS that is equivalent to a $34 \%$ increase in risk of 12 month mortality per 10-point (1 SD) decrement in PCS scores. The MCS was a significant predictor of mortality after adjusting for demographic and cardiac risk factors $(\mathrm{OR}=0.96, \mathrm{CI}=0.93-0.99, P=.010)$. When symptoms of

\section{Table 2}

Results from hierarchical logistic regression analyses predicting mortality using SF-12 PCS score with and without adjustment for other prognostic factors

\begin{tabular}{lllr}
\hline & & \multicolumn{2}{l}{$95 \%$ Confidence } \\
Variable & Odds ratio & interval & $P$ value \\
\hline PCS & & & \\
$\quad$ SF-12 PCS Score & 0.94 & $0.92-0.97$ & $<.001$ \\
PCS+demographic and & & & \\
$\quad$ cardiac prognostic factors & & & \\
SF-12 PCS Score & 0.95 & $0.92-0.98$ & $<.001$ \\
Age & 1.05 & $1.02-1.08$ & .004 \\
Female sex & 0.49 & $0.24-1.02$ & .055 \\
Diagnosis of AMI (vs. UA) & 1.47 & $0.76-2.83$ & .252 \\
Previous MI & 2.23 & $1.17-4.25$ & .015 \\
Killip class >1 & 1.20 & $0.54-2.48$ & .642 \\
PCS+demographic and cardiac & & & \\
prognostic factors+depressive & & & \\
$\quad$ symptoms & & & \\
SF-12 PCS Score & 0.96 & $0.93-0.99$ & .008 \\
Age & 1.06 & $1.03-1.10$ & $<.001$ \\
Female sex & 0.39 & $0.18-0.82$ & .013 \\
Diagnosis of AMI (vs. UA) & 1.63 & $0.84-3.17$ & .151 \\
Previous MI & 2.09 & $1.09-4.01$ & .027 \\
Killip class >1 & 1.03 & $0.47-2.22$ & .951 \\
BDI Total score & 1.07 & $1.03-1.11$ & .001 \\
\hline
\end{tabular}

For the SF-12 PCS score, a higher score reflects better function. For the $\mathrm{BDI}$, a higher score reflects greater depressive symptomatology.
Table 3

Results from hierarchical logistic regression analyses predicting mortality using SF-12 MCS score with and without adjustment for other prognostic factors

\begin{tabular}{lllr}
\hline Variable & $\begin{array}{l}\text { Odds } \\
\text { ratio }\end{array}$ & $\begin{array}{l}95 \% \text { Confidence } \\
\text { interval }\end{array}$ & $P$ value \\
\hline MCS & & & \\
SF-12 MCS Score & 0.98 & $0.95-1.00$ & .053 \\
MCS+demographic and & & & \\
$\quad$ cardiac prognostic factors & & & \\
SF-12 MCS Score & 0.96 & $0.93-0.99$ & .010 \\
Age & 1.06 & $1.03-1.10$ & $<.001$ \\
Female sex & 0.52 & $0.25-1.07$ & .075 \\
Diagnosis of AMI (vs. UA) & 1.25 & $0.66-2.36$ & .503 \\
Previous MI & 2.56 & $1.35-4.82$ & .004 \\
Killip class $>1$ & 1.40 & $0.66-2.96$ & .384 \\
MCS+demographic and cardiac & & & \\
$\quad$ prognostic factors+depressive & & & \\
symptoms & & & .534 \\
SF-12 MCS Score & 0.99 & $0.96-1.02$ & $<.001$ \\
Age & 1.07 & $1.04-1.11$ & .027 \\
Female sex & 0.43 & $0.20-0.91$ & .338 \\
Diagnosis of AMI (vs. UA) & 1.38 & $0.72-2.64$ & .009 \\
Previous MI & 2.37 & $1.25-4.52$ & .668 \\
Killip class >1 & 1.18 & $0.55-2.53$ & .003 \\
BDI Total score & 1.07 & $1.02-1.12$ & \\
\hline
\end{tabular}

For the SF-12 MCS score, a higher score reflects better function. For the BDI, a higher score reflects greater depressive symptomatology.

depression were included in the model, higher symptoms of depression were significantly associated with greater mortality risk $(\mathrm{OR}=1.07, \mathrm{CI}=1.02-1.12, P=.003)$, but the $\mathrm{SF}-12$ MCS was not $(\mathrm{OR}=0.99, \mathrm{CI}=0.96-1.02, P=.534)$. In addition, age and history of MI significantly predicted mortality in both regression models. Sex was a significant predictor in some, but not all, models. The final models for both PCS and MCS had good discriminative power (cindex $=.79$ and .77 , respectively) and calibration ( $P=.424$ and $P=.423$ for HL statistic, respectively). Post hoc analysis with additional variables from Table 1 included as covariates did not alter findings related to the PCS or MCS.

\section{Discussion}

The main finding of this study was that physical health status assessed during hospitalization for AMI or UA independently predicted mortality 1 year later even after adjusting for traditional clinical risk factors and symptoms of depression. A decrease of 10 points (1 SD in the normative sample) in PCS scores on the SF-12 was associated with a $34 \%$ higher risk of mortality 12 months after an ACS. In-hospital MCS scores, on the other hand, tended to predict mortality in bivariate analysis (nonsignificant), but were not a factor in multivariate analysis after controlling for risk factors, including depressive symptoms. The relationship of symptoms of depression with mortality was significant when included in prognostic models with the PCS or the MCS, and each 1-point increase in total BDI 
score was associated with a $7 \%$ higher risk of death. Thus, in addition to patient-reported physical health, patientreported mental health as assessed by the BDI, but not the MCS, predicted 12-month mortality.

The finding that the PCS, but not the MCS, predicts mortality is consistent with the results of studies of patients undergoing CABG surgery [4] and hospitalized heart failure patients [14], in which the SF-36 PCS, but not the MCS, predicted mortality approximately 6 months after hospital discharge. Studies of heart failure patients that have used other measures of self-reported health status, such as the Minnesota Living with Heart Failure Questionnaire, the Seattle Angina Questionnaire, or the Nottingham Health Profile, have generally found that global and/or physical health status scores significantly predict mortality in multivariate analyses, but that the relationship between mental or emotional health and mortality varies across studies [10-15]. One recent study reported that after controlling for cardiac risk factors and symptoms of depression, mortality was not related to the SF-36 PCS, the SF-36 MCS, or the clinical summary score of the Kansas City Cardiomyopathy Questionnaire. The authors attributed this finding to the robust prognostic power of the New York Heart Association functional class variable, although it is possible that relatively small sample size was also a factor $(N=231)$ [16].

It is notable in the present study that the SF-12 MCS was not a significant predictor of mortality, whereas the BDI was a significant and strong predictor of mortality. This may relate to how the SF-12 summary scores are calculated and, specifically, to the use of negative scoring coefficients to calculate the summary scores. The scoring methods for calculating the PCS and MCS summary scores of the SF-36 and SF-12 were designed to create orthogonal (uncorrelated) factor scores in order to eliminate the problem of multicollinearity when using both component scores in multiple regression. To achieve this, low scores on some of the physically oriented items (poor functioning) are scored negatively on the MCS, resulting in better mental function scores. The opposite is also true. Farivar et al. [30] recently demonstrated that when physical subscale scores are well below the mean and mental subscale scores somewhat less below the mean, as is often the case in patients with medical illness, this scoring method will result in an artifactual migration of the aggregate PCS score away from the mean and a migration of the aggregate MCS score towards the mean. This phenomenon can be illustrated by comparing SF36 physical subscale scores with the PCS and SF- 36 mental subscale scores with the MCS; the study of hospitalized heart failure patients by Rodriguez-Artalejo et al. [14] provides such data. In that study, patient scores on the physical subscales (physical functioning, physical role, bodily pain, general health perception) were 0.3 to 2.0 SDs (mean 1.3 SDs) lower than the US general population (http://www. sf-36.org/nbscalc/index.shtml), and the PCS score was approximately 1.6 SDs below the general population mean. Scores on the mental subscales (mental health, emotional role, vitality, social functioning) were 0.7 to 1.2 SDs lower than the US general population scores (mean $0.9 \mathrm{SDs}$ ), but the MCS was only 0.6 SDs lower than the US population standardization sample mean. An important implication of this scoring artifact is that relationships between mental health factors and outcomes of interest may be dampened sufficiently when combined in the aggregate scores to not be detected [31]. Although this phenomenon is less easily illustrated for the SF-12 aggregate scores, it is similarly present. Another possible reason is that the 21 -item $\mathrm{BDI}$ was a more reliable predictor than the shorter SF-12 MCS.

Thus, both the BDI and the SF-12 PCS were useful in predicting mortality at 12 months and would be useful risk stratification tools in the post-ACS setting. Depression is a well-known risk factor for post-ACS mortality and is associated with functional impairment [32], less favorable self-care behaviors [33], higher health care costs [34], and increased cardiac morbidity and mortality $[18,35]$. Self-rated physical health may quantify underlying aspects of health not captured by traditional indicators of disease severity or it may serve as a proxy when these indicators are not available. Physical health may also quantify important aspects of health not related to cardiovascular disease. In terms of prevention and rehabilitation, this study shows that a broad approach to conceptualizing post-ACS health may be useful for understanding risk. Although the BDI and PCS may both be useful for risk stratification, this study does not provide sufficient data to recommend that either be used as part of the routine care of ACS patients. As demonstrated in primary care settings for depression, improved outcomes from depression screening are dependent upon the use of integrative care models including the use of evidence-based protocols for treatment, active collaboration between primary care providers and mental health specialists, active monitoring of adherence to therapy, and access to structured psychotherapy [36-38]. Thus far, no studies have tested whether screening for depression or self-rated health status benefits patients in cardiovascular care settings.

A limitation of this study was that mortality was assessed only at 12 months post-ACS, which did not provide time to death data. Thus, logistic regression was conducted, rather than survival analysis, which could have affected results. The 12-month assessment point was arbitrary, and it is not known whether the results produced by the regression model at 12 months would have been the same at other time points (e.g., 24 or 36 months). Mortality data did not include whether or not death was cardiac related and was done via report of patients' family members, general practitioners, or specialist physicians, the accuracy of which has not been demonstrated. In addition, there were a relatively small number of deaths, and it is not known whether the risk estimates generated from this sample would be replicated in a larger sample with more deaths. The small number of deaths may also explain why Killip class, for instance, which has been strongly and consistently related to mortality risk factor in previous studies [25-28], showed a trend towards 
higher mortality in bivariate analysis, but did not reach statistical significance. An additional limitation was that information was not available on several important risk markers, including left ventricular ejection fraction, renal function, revascularization procedures, or medications used. Thus, it is possible that our results may reflect some degree of residual confounding.

In conclusion, the PCS of the SF-12 presents an attractive option for improving risk stratification since it significantly predicts mortality and may detect aspects of physical health and mortality risk that may not be captured by more commonly used indicators of cardiac disease severity or by other traditional risk factors. The association of self-rated physical health should be tested in future studies that control for a broader spectrum of risk factors than were available in this study.

\section{Acknowledgments}

The authors are grateful to Linda Green for her diligence in study coordination.

\section{References}

[1] Rumsfeld JS. Health status and clinical practice: when will they meet? Circulation 2002;106:5-7.

[2] Bren $L$. The importance of patient-reported outcomes...it's all about the patients. FDA Consum 2006;40:26-32.

[3] Cella D, Yount S, Rothrock N, Gershon R, Cook K, Reeve B, et al, The Patient-Reported Outcomes Measurement Information System (PROMIS): progress of an NIH Roadmap cooperative group during its first two years. Med Care 2007;45:S3-SI 1.

[4] Rumsfeld JS, MaWhinney S, McCarthy M, Shroyer AL, VillaNueva $\mathrm{CB}, \mathrm{O}$ 'Brien $\mathrm{M}$, et al. Health-related quality of life as a predictor of mortality following coronary artery bypass graft surgery. Participants of the Department of Veterans Affairs Cooperative Study Group on Processes, Structures, and Outcomes of Care in Cardiac Surgery. JAMA 1999;281:1298-303.

[5] Rumsfeld JS, Ho PM. Depression and cardiovascular disease: a call for recognition. Circulation 2005;111:250-3.

[6] Ware JE, Kosinski M, Keller SD. SF-36 physical and mental health summary scales: a user's manual. Boston (Mass): The Health Institute, New England Medical Center, 1994.

[7] Ware J, Kosinski M, Keller SD. A 12-Item Short-Form Health Survey: construction of scales and preliminary tests of reliability and validity. Med Care 1996;34:220-33.

[8] Myint PK, Luben RN, Surtees PG, Wainwright NW, Welch AA, Bingham SA, et al. Relation between self-reported physical functional health and chronic disease mortality in men and women in the European Prospective Investigation into Cancer (EPICNorfolk): a prospective population study. Ann Epidemiol 2006;16: 492-500.

[9] Spertus JA, Jones P, McDonell M, Fan V, Fihn SD. Health status predicts long-term outcome in outpatients with coronary disease. Circulation 2002;106:43-9.

[10] Konstam V, Salem D, Pouleur H, Kostis J, Gorkin L, Shumaker S, et al. Baseline quality of life as a predictor of mortality and hospitalization in 5,025 patients with congestive heart failure. SOLVD Investigations. Studies of Left Ventricular Dysfunction Investigators. Am J Cardiol 1996;78:890-5.

[11] Alla F, Briancon S, Guillemin F, Juilliere Y, Mertes PM, Villemot JP, et al. Self-rating of quality of life provides additional prognostic information in heart failure. Insights into the EPICAL study. Eur J Heart Fail 2002;4:337-43.

[12] Mejhert M, Kahan T, Persson H, Edner M. Predicting readmissions and cardiovascular events in heart failure patients. Int J Cardiol 2006;109: 108-13.

[13] Bouvy ML, Heerdink ER, Leufkens HG, Hoes AW. Predicting mortality in patients with heart failure: a pragmatic approach. Heart 2003;89:605-9.

[14] Rodriguez-Artalejo F, Guallar-Castillon P, Pascual CR, Otero CM, Montes AO, Garcia AN, et al. Health-related quality of life as a predictor of hospital readmission and death among patients with heart failure. Arch Intern Med 2005;165:1274-9.

[15] Soto GE, Jones P, Weintraub WS, Krumholz HM, Spertus JA. Prognostic value of health status in patients with heart failure after acute myocardial infarction. Circulation 2004;110:546-51.

[16] Faller H, Stork S, Schowalter M, Steinbuchel T, Wollner V, Ertl G, et al. Is health-related quality of life an independent predictor of survival in patients with chronic heart failure? J Psychosom Res 2007; 63:533-8.

[17] Murberg TA, Furze G. Depressive symptoms and mortality in patients with congestive heart failure: a six-year follow-up study. Med Sci Monit 2004;10:CR643-8.

[18] van Melle JP, de Jonge P, Spijkerman TA, Tijssen JG, Ormel J, van Veldhuisen DJ, et al. Prognostic association of depression following myocardial infarction with mortality and cardiovascular events: a metaanalysis. Psychosom Med 2004;66:814-22.

[19] Sorensen C, Friis-Hasche E, Haghfelt T, Bech P. Postmyocardial infarction mortality in relation to depression: a systematic critical review. Psychother Psychosom 2005;74:69-80.

[20] Lesperance F, Frasure-Smith N, Juneau M, Theroux P. Depression and 1-year prognosis in unstable angina. Arch Intern Med 2000;160: $1354-60$.

[21] Grech ED, Ramsdale DR. Acute coronary syndrome: unstable angina and non-ST elevation myocardial infarction. BMJ 2003;326: 1259-61.

[22] Muller-Nordhorn J, Roll S, Willich SN. Comparison of the short form (SF)-12 health status instrument with the SF-36 in patients with coronary heart disease. Heart 2004;90:523-7.

[23] Beck AT, Steer RA. Manual for the Revised Beck Depression Inventory. San Antonio (Tex): Psychological Corporation, 1987.

[24] Thombs BD, Bass EB, Ford DE, Stewart KJ, Tsilidis KK, Patel U, et al. Prevalence of depression in survivors of acute myocardial infarction. J Gen Intern Med 2006;21:30-8.

[25] Killip T, Kimball JT. Treatment of myocardial infarction in a coronary care unit. A two year experience with 250 patients. Am J Cardiol 1967; 20:457-64.

[26] Khot UN, Jia G, Moliterno DJ, Lincoff AM, Khot MB, Harrington RA, et al. Prognostic importance of physical examination for heart failure in non-ST-elevation acute coronary syndromes: the enduring value of Killip classification. JAMA 2003;290:2174-81.

[27] Steg PG, Dabbous OH, Feldman LJ, Cohen-Solal A, Aumont MC, Lopez-Sendon J, et al. Determinants and prognostic impact of heart failure complicating acute coronary syndromes: observations from the Global Registry of Acute Coronary Events (GRACE). Circulation 2004;109:494-9.

[28] Segev A, Strauss BH, Tan M, Mendelsohn AA, Lai K, Ashton T, et al. Prognostic significance of admission heart failure in patients with nonST-elevation acute coronary syndromes (from the Canadian Acute Coronary Syndrome Registries). Am J Cardiol 2006;98:470-3.

[29] Hosmer DW, Lemeshow S. Applied Logistic Regression. 2nd ed. New York: John Wiley \& Sons, 2000.

[30] Farivar SS, Cunningham WE, Hays RD. Correlated physical and mental health summary scores for the SF-36 and SF-12 Health Survey, V.I. Health Qual Life Outcomes 2007;5:54.

[31] Simon GE, Revicki DA, Grothaus L, Vonkorff M. SF-36 summary scores: are physical and mental health truly distinct? Med Care 1998; $36: 567-72$ 
[32] Ruo B, Rumsfeld JS, Hlatky MA, Liu H, Browner WS, Whooley MA. Depressive symptoms and health-related quality of life: the Heart and Soul Study. JAMA 2003;290:215-21.

[33] Ziegelstein RC, Fauerbach JA, Stevens SS, Romanelli J, Richter DP, Bush DE. Patients with depression are less likely to follow recommendations to reduce cardiac risk during recovery from a myocardial infarction. Arch Intern Med 2000;160:1818-23.

[34] Frasure-Smith N, Lesperance F, Gravel G, Masson A, Juneau $M$, Talajic M, et al. Depression and health-care costs during the first year following myocardial infarction. J Psychosom Res 2000;48:471-8.
[35] Barth J, Schumacher M, Herrmann-Lingen C. Depression as a risk factor for mortality in patients with coronary heart disease: a metaanalysis. Psychosom Med 2004;66:802-13.

[36] U.S. Preventive Services Task Force. Screening for depression: recommendations and rationale. Ann Intern Med 2002;136: $760-4$.

[37] Stewart DE. Battling depression. CMAJ 2008;178:1023-4.

[38] Gilbody S, Sheldon T, House A. Screening and case-finding instruments for depression: a meta-analysis. CMAJ 2008;178: 997-1003. 